\title{
REDES SOCIALES: TRANSFORMANDO LA COMUNICACIÓN EN LAS RELACIONES INTERPERSONALES
}

\author{
Dulce María Guillén Cadena y Vianey Reyes García \\ UNAM \\ México
}

\begin{abstract}
RESUMEN
Introducción: Las relaciones interpersonales, es decir, la relación y comunicación que se establece entre dos o más personas se encuentra en constante transformación en la cotidianidad del ser humano, y en esta transformación han incidido las nuevas aplicaciones tecnológicas generando cambios en la comunicación interpersonal. Objetivo: Interpretar cómo se ha modificado la comunicación en las relaciones interpersonales a partir de la aparición de las redes sociales en trabajadores administrativos entre 29 y 35 años de edad, de una tienda departamental. Metodología: Cualitativa, fenomenológica interpretativa, la recolección de los datos se obtuvo por medio de la entrevista a profundidad, observación y notas de campo. Hallazgos: La comunicación tradicional ha sufrido cambios en los elementos clave de la misma, es poco afectiva, carente de emociones, pobre en el lenguaje etcétera. Conclusión: El uso del móvil y redes sociales desde una perspectiva utópica permite mantener relaciones interpersonales donde la distancia y el tiempo no lo permiten; sin embargo, esta cualidad afecta las relaciones interpersonales cercanas.
\end{abstract}

Palabras Clave:

redes sociales, comunicación, relaciones interpersonales

\section{SOCIAL NETWORKS: TRANSFORMING COMMUNICATION INTO INTERPERSONAL RELATIONSHIPS}

\begin{abstract}
Introduction: The interpersonal relationships are in constant transformation in the daily life of the human being, and in this transformation have influenced the new technological applications generating changes in interpersonal communication. Objective: Interpret how communication has been modified in interpersonal relationships from the appearance of social networks in administrative workers of a departmental store. Methodology: Qualitative, interpretive phenomenological, data collection was obtained through in-depth interview, observation and field notes. Findings: Traditional communication has undergone changes in the key elements of the same, is little affective, lacking in emotions, poor in language etc. Conclusion: The use of mobile phones and social networks from a utopian perspective allows maintaining interpersonal relationships where distance and time do not allow it, however this quality affects the close interpersonal relationships.
\end{abstract}

Keywords:

students, high school, friendship, companionship, gender

Bitácora del ARtículo:

| Recibido: 25 de Septiembre de 2018 | Aceptado: 4 de Abril de 2019 | Publicado en línea: Julio - Diciembre de 2019 | 
Artículo Empírico | Redes sociales: Transformando la comunicación... | Guillén-Cadena eq Reyes-García

\title{
Autoría y Derechos de Propiedad Intelectual
}

\section{REDES SOCIALES: TRANSFORMANDO LA COMUNICACIÓN EN LAS RELACIONES INTERPERSONALES}

\author{
Dulce María Guillén Cadena y Vianey Reyes García
}

UNAM

México

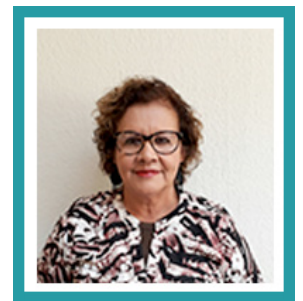

Dulce María Guillén Cadena

FES Iztacala, UNAM

Correo: dulce1414@gmail.com

Lic. enfermera con especialidad en docencia y psiquiatría; maestría en modificación de conducta; doctorada en educación; profesora titular " $C$ " Carrera de Enfermería; responsable del Taller permanente de Titulación; fundadora y directora de la revista Cuidarte; responsable del semillero de investigación, profesora del módulo de metodología de investigación, Profesora y tutora de la maestría en Ciencias de la Enfermería.

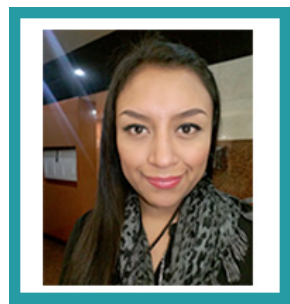

Vianey Reyes García FES Iztacala, UNAM

Correo: vianey.reyes.vi@gmail.com

Licenciada en enfermería, maestranda del programa de maestría en enfermería en Unidad de Posgrado UNAM, becaria Conacyt, con experiencia en enfermería ocupacional y coordinación de seguridad e higiene en centro de distribución. Corresponsable en línea de investigación Tecnologías y Salud, liderada por la doctora Dulce María Guillén Cadena; asistente editorial en revista CuidArte "El Arte del Cuidado".

\section{CONTRIBUCIÓN DE LOS AUTORES}

La primera autora desarrollo y coordinación del proyecto, análisis de datos y redacción de los hallazgos, conclusiones. | La segunda autora realizo la recolección de la información, análisis y redacción de los hallazgos

\section{AGRADECIMIENTOS}

Agradecemos la colaboración de nuestros participantes, quien a través de su apoyo hicieron posible el desarrollo de la investigación.

\section{DATOS DE FILIACIÓN DE LOS AUtORES}

Facultad de Estudios Superiores Iztacala UNAM

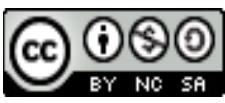

Copyright: (c) 2019 Guillén-Cadena, D. M. \& Reyes-García, V.

Este es un artículo de acceso abierto distribuido bajo los términos de la licencia Creative Commons Reconocimiento-NoComercial 4.0 Internacional, por lo que su contenido gráfico y escrito se puede compartir, copiar y redistribuir total o parcialmente sin necesidad de permiso expreso de sus autoras con la única condición de que no se puede usar con fines directamente comerciales y los términos legales de cualquier trabajo derivado deben ser los mismos que se expresan en la presente declaración. La única condición es que se cite la fuente con referencia a la Revista Digital Internacional de Psicología y Ciencia Social y a sus autoras. 


\section{TABLA DE CONTENIDO}

INTRODUCCIÓN

MÉTODO

Objetivo General, 34

Participantes (típico ideal), 34

Procedimiento, 34

Diseño, 34

Aspectos éticos, 34

Criterios de rigor, 34

Análisis estadísticos, 34

RESULTADOS Y DISCUSIÓN

Comunicación, 35

Rol familiar, 35

Ruptura en las relaciones familiares, 36

Cambios en la dinámica, 36

CONCLUSIONES

Sugerencias y limitaciones, 37

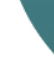




\section{INTRODUCCIÓN}

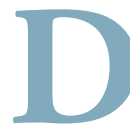
esde una perspectiva estadística, el uso del teléfono móvil marca un crecimiento exponencial, con $66 \%$ de inserción a nivel mundial, en paralelo al internet con una penetración de $50 \%$, generando una transformación frente a la comunicación y las relaciones interpersonales. Basta con ver las cifras de los hábitos de consumo para entender la nueva realidad digital; análisis estadísticos indican que $61 \%$ de los usuarios del móvil en el mundo afirma observarlo dentro de los primeros cinco minutos después de despertarse, pasando una media de 170 minutos al día, siendo la consulta de redes sociales, navegación de internet y visualización de videos la mayoría de actividades efectuadas desde el mismo. De este modo, por medio de sus aplicaciones el teléfono móvil se ha convertido en el dispositivo favorito de una nueva generación, nacida a principios de los años ochenta del siglo anterior y los primeros de este siglo; los millenials, caracterizados por su familiarización con los términos tecnológicos, al formar parte de la consolidación de una nueva comunicación mediante el internet y este dispositivo, y quienes dedican en promedio 93.5 horas al mes utilizando las mañanas para leer mails, mediodía para chatear, tarde para utilizar apps de movilidad, compras (además de chatear) y la noche para las redes sociales, por lo que las apps de comunicaciones y redes sociales son las más utilizadas, seguidas de otras aplicaciones como negocios, finanzas etcétera (Rivero, 2017).

Son las redes sociales las que han dado un nuevo giro a la manera de interacción entre las personas, contando en el mundo con una inserción de $34 \%$ respecto a la población, $7 \%$ más que en 2016. Facebook encabeza la lista con 1.493 millones de usuarios, Instagram con 400 millones. Redes a las que acceden casi $98 \%$ de los usuarios desde su móvil (Rivero, 2017).

En México las cosas también están cambiando; en su tercera edición el Global Mobile Consumer Survey (GMCS), encuesta enfocada en los comportamientos, tendencias y opiniones de los consumidores de las tecnologías de la información y comunicación (TIC), lanzó datos interesantes acerca del uso del teléfono móvil en la población mexicana, destacando que $83 \%$ revisa su teléfono en los primeros 30 minutos, sobre todo para acceder a redes sociales, mensajes instantáneos y correo, además de indicar que casi $79 \%$ en una edad entre los 18-24 años revisa el teléfono en la madrugada, con $65 \%$ de los casos entre las personas de 25 a 44 años de edad (Ortiz, Hegewisch, Palacios y Espinoza, 2016). Sin dejar de lado los datos que ofrece la asociación de internet en México, que indica - por medio de su 13o. Informe-que los internautas pasan en promedio 8 horas con 1 minuto y 47 segundos de tiempo de conexión, haciéndolo $90 \%$ desde su teléfono móvil, siendo $83 \%$ de los casos para acceder a alguna red social (Tamayo, 2017).

De este modo, la relación del teléfono móvil y la diversificación de su uso va más allá de su empleo para llamadas, haciéndolo un dispositivo muy adictivo, con una penetración demandante a nivel mundial, y por tanto en la sociedad y su contexto. Su utilidad es cada vez más profunda, porque por medio de él se consolidan varios aspectos de nuestras vidas (Ortiz et al., 2016) al imponer modas, ser fuente de identidad, sustituir otras tecnologías como la cámara, agenda, reloj, calculadora y reproductor de música, entre muchas otras (Luz, 2010).

Evidencia así una dualidad entre sus cualidades al obtener satisfacción respecto a su uso, pero con la desventaja de ser adictivo, categorizándolo así en las adicciones comportamentales, es decir las sin sustancia, pero que igual tienen síntomas como falta de control y dependencia (Pedrero, Rodríguez y Ruiz 2012), además de presentarse cambios físicos por su uso frecuente, como síndrome del túnel carpiano (presión en el nervio de la muñeca, que genera entumecimiento, debilidad y/o daño muscular), alteraciones de la agudeza visual, nomofobia (miedo a no traer consigo el teléfono móvil), portalitis (molestias y dolores musculares) e incluso lesiones irreversibles a nivel del sistema nervioso central (Guillén, Beltrán, Flores, Aristizabal y Garduño-Magaña, 2016).

Hoy no hay ninguna dimensión en la vida que se excluya de la transformación generada por las tecnologías, la extensión de las TIC a todas los niveles sociales depende de diferentes aspectos: infraestructuras, capacidades, elementos familiares y de la vida cotidiana, entre otros, que pueden o no facilitar la apropiación de las nuevas tecnologías. Las redes sociales son un fenómeno tecnológico de reciente aparición, que influye de modo social y que permea en las nuevas "formas de interacción", proponiendo una dinámica diferente en las relaciones entre personas, grupos e instituciones (Cornejo y Tapia, 2012).

Las relaciones interpersonales están en constante transformación en la cotidianidad del ser humano, y en esta transformación han incidido las nuevas aplicaciones tecnológicas que generan cambios en la comunicación interpersonal. El que las interacciones digitales entre las personas se incrementen tanto crea una percepción distinta del espacio y el tiempo, una sensación de inmediatez de los acontecimientos y una aceleración en los procesos. 
Son precisamente las relaciones interpersonales las que se ven afectadas, la trasmisión de emociones y sentimientos se limita, porque no se da cara a cara, no se observan gestos, expresión facial, mirada, tono de voz, énfasis, movimientos de las manos, etcétera.

\section{MÉTodo}

\section{Objetivo General}

Interpretar cómo se ha modificado la comunicación en las relaciones interpersonales a partir de la aparición de las redes sociales en trabajadores administrativos del centro de distribución de una tienda departamental.

\section{Participantes (típico ideal)}

La selección de los informantes se hizo de acuerdo con el típico ideal (Rodríguez, Gil y García, 1999): personas que mantuvieran un uso continuo del teléfono móvil y se encontraran laborando; participaron tres mujeres y un hombre con un rango de edad entre los 29 y 35 años, con nivel de estudios de licenciatura y maestría.

\section{Procedimiento}

La recolección de los datos se efectuó por medio de la entrevista a profundidad, la cual inició con una pregunta detonante; tuvo una duración entre 45 a 60 minutos; las conversaciones fueron grabadas en una magnetofónica previo consentimiento informado. Se llevó a cabo en las instalaciones del centro de distribución de una reconocida empresa departamental de lujo; también se usaron la observación participante y notas de campo que se hicieron conforme transcurrió la entrevista.

\section{Diseño}

Esta investigación se efectuó con una metodología cualitativa, con diseño fenomenológico interpretativo, que permitió enfocarnos en la experiencia personal de cada uno de los informantes, dando búsqueda a los significados posibles (Álvarez-Gayou, 2005).

\section{Aspectos éticos}

Se tuvieron en cuenta los principios éticos básicos del Informe Belmont (Comisión Nacional de Bioética, 1979) sumando el valor social en relación con la importancia de esta investigación, con validez científica, selección equitativa de los sujetos y de respeto y evaluación independiente (Koepsell y Ruiz, 2015), así como la aplicación del consentimiento informado en quienes aceptaron participar y cuyo consentimiento fue grabado momentos previos de iniciar la entrevista

\section{Criterios de rigor}

Los criterios de rigor a considerar fueron los propuestos por Castillo (2000): credibilidad, permitiendo captar lo cierto en relación con la situación social que vivían, y poder partir de una reflexión; por otro lado auditabilidad, con base en la determinación que hacen los autores para la técnica de recolección de datos por medio de la utilización de una grabadora magnetofónica y la transcripción de las entrevistas y la transferibilidad, porque puede ser aplicada en otro contexto.

\section{Análisis estadísticos}

El análisis sistemático se hizo de acuerdo con lo propuesto por Krueger: lectura y relectura de las entrevistas, transcripción de las mismas con un procesador de textos Microsotf Word 2016; se codificaron con plumines de colores y se establecieron los temas y subtemas.

Es muy importante abordar este tema desde una perspectiva cualitativa, porque las características de la misma permiten hacer el análisis y encontrar la explicación flexible y sensible del entrevistado, así como comprender el contexto social en el cual se desarrolla, partiendo así de una perspectiva holística imprescindible en esta metodología; con la misma fue posible conjugar todas las influencias para que las personas hagan uso consciente del teléfono móvil.

Para comprender el significado del uso del teléfono móvil y sus aplicaciones en los entrevistados, retomamos a Jerome Bruner para clarificar el fenómeno a estudiar, permitiéndonos así conocer las atribuciones que cada uno de ellos le otorgó a partir de la construcción de su mundo interno, entre sus creencias y deseos del mundo de afuera, que es la cultura; también nos permitió visualizar cómo se han modificado sus relaciones interpersonales.

\section{Resultados y discusión}

El análisis sistemático del cual se habló en la metodología, desglosó una serie de temas y subtemas a partir de los hallazgos obtenidos por medio del discurso de los informantes, permitiendo reconocer en primera instancia alteraciones en la comunicación, la cual se tornó poco afectiva, despersonalizada y pobre en el lenguaje, lo cual a su vez generó una alteración en el rol familiar, desencadenando ruptura de las relaciones familiares y cambios en la dinámica de la misma. 


\section{Comunicación}

La transformación en la comunicación cada vez se despersonaliza más, generando nuevos modos de interacción verbal que se distancia mucho de la cercanía, presencia y atención que exigía en su momento la comunicación tradicional. Ahora está Ilena de obstáculos que reducen el contacto y dejan un vacío de calidez, al transmitirse sólo mediante una pantalla, envío y recepción de mensajes prácticos e indirectos.

Lojo (2014) se refiere a esta comunicación entre pares teniendo como intermediario a un dispositivo que exige una nueva escritura y un nuevo lenguaje; una deconstrucción del lenguaje en esta sociedad ávida de consumo y tecnología construyendo entonces mundos virtuales, escribiendo en soledad y leyendo en soledad sin ningún contacto inmediato (Fortunati y Magnanelli, 2002).

En voz de los entrevistados:

[...] Lo que platicamos, ya no lo platicamos de frente; ya lo platicamos por el celular, y cuando nos vemos ya es así como de aaaa... ¿y luego?

[...] La comunicación que antes se manejaba por una llamada o face to face... es suplida por el celular o un whats app; o sea, es más fácil que esperes un whats app de alguien, que una llamada.

\section{Comunicación poco afectiva}

Son los mensajes indirectos que se envían a través de redes sociales, sobre todo con el uso de la aplicación de Whatsapp; dichos mensajes generan deficientes entendidos en la comunicación, haciendo que se pierdan registros como la mirada y el lenguaje corporal, lo que modifica la verbalización por el "texteo" (Giménez y Zirpoli, 2015).

Ahora son las redes sociales las que moldean el lenguaje y precisan que los mensajes sean rápidos antes que claros y sintéticos antes que explicativos, sin importar cómo se escriba mientras se entiendan, con lo que se recrea una informalidad en la comunicación interpersonal (Lojo, 2014).

En voz de los entrevistados:

[...] Ya no hay comunicación visual.

[...] A veces no muchas personas tienen [...] como la fortaleza de decir las cosas de cara a la gente; entonces se esconden detrás de [...] un dispositivo [...] donde puedes agarrar más valor de decir cosas que no dirías a través de una llamada telefónica que cuando tienes a la persona de frente.
[...] A través de los textos no se transfieren los sentimientos o las emociones, ¿no?, que seguramente cuando estés frente alguien puedes transmitirle, y pues la otra contraparte es que a veces lo que yo intente decir en el texto [...] pues tal vez la persona, el receptor, no lo va a entender de la misma forma que yo esperaría.

\section{Comunicación despersonalizada}

Permanecer y entablar una plática cada vez es más difícil, con lo que se genera que sólo exista la presencia fisca y no mental, denominada como "desconectarse mentalmente" (Richard, 2004), ignorando el aquí y ahora, perdiéndose la oportunidad de comunicarse con la persona que tenemos enfrente, compartir cualquier experiencia se complica, cayendo en un ocio permisivo e individualista (Aparicio y Zermeño, 2010).

En voz de los entrevistados:

[...] Por ejemplo, yo tengo a mi compañera con la que como y estamos comiendo, y de repente estoy hablando y estoy platicando, pero ella [simula ver un celular], y yo, así como de qué onda; o sea, ella con el celular y yo hablando y platicando [...] es más fácil que me conteste por el Whatsapp que de frente; así, platicando, ya es difícil.

[...] A veces me pierdo un poquito de esa parte de interactuar con las personas, por meterme en el teléfono.

\section{Rol familiar}

Manifestándose así estos cambios en el rol familiar y/o pareja y su dinámica por medio de la interacción que había entre los integrantes. Supliendo encuentros a la hora de comer juntos, reuniones familiares por permanecer detrás de una pantalla con un "teléfono inteligente", con el uso de redes sociales y aplicaciones, tomando y mediando espacios considerables en la familia, y por tanto la relación entre los integrantes (Aparicio y Zermeño, 2010).

En voz de los entrevistados:

[...] En casa de diez, siete estamos viendo el teléfono [...] le das más importancia a otras cosas que, pues a la convivencia familiar.

[...] Con mi familia, Ilamémoslo en cuestión de que llego a estar con mi familia, si no lo tengo obviamente convives más [...] O sea, todo mundo se dedica a estar como revisando el teléfono.

[...] La relación con los amigos [...] sólo es por la red social, con el móvil. 
[...] Últimamente damos más importancia a estar con el aparato que con la familia.

[...] Llegaba, platicaba, veíamos la tele; salíamos y cosas así; ahora me la paso encerrada en mi cuarto [...] estoy pegada en el teléfono.

\section{Ruptura en las relaciones familiares}

Considerando las características del teléfono móvil como el principal factor de distracción entre los miembros de una familia, y por tanto de discusión al hacer uso de él en lugares donde no se debe; al convivir, platicar o compartir un momento que para los receptores es importante (Antolinez, Rivero y Torres, 2013).

En voz de los entrevistados:

[...] vivo con mis padres [...] que de repente me dicen así de "Oye, estás comiendo; estás cenando con nosotros, así que pon atención, deja el teléfono por lo menos cuando estás comiendo, ¿no? [...] no te vemos todo el día, llegas en la noche y estás con el teléfono [...] dedícanos tiempo [...] algunos momentos si te molesta".

[...] Es algo que me dice a lo mejor mi pareja, mi mamá, "ya deja ese teléfono" [...] porque yo antes no era así.

[...] Problemas con la pareja [...] Si muchos; actualmente trato de esconderlo, de hacerlo a un lado; pero aun así se queda como mi cabeza pensando "chin lo deje ahí, ¿y si llegan mensajes, ¿y si algo está pasando y corro a verlo no?"; sí, lo he intentado, pero cuesta muchísimo trabajo.

[...] De pronto con mi pareja sí he tenido algunas broncas porque hay ocasiones que él sí me ha tenido que pedir muchas veces, cuando estamos comiendo, "deja el celular".

\section{Cambios en la dinámica}

La disponibilidad del teléfono móvil hace a las personas localizables sin importar el tiempo y el espacio; en el plano familiar y de las relaciones interpersonales se muestra bilateral, siendo hasta cierto punto útil para la localización y comunicación cercana con familiares que tenemos lejos, pero que una vez más nos aproxima al control y disponibilidad que se debe tener para con los demás, traspasando incluso nuestra individualidad, privacidad y disponibilidad a todas horas; creen tan sólo en Whatsapp que permanecer en línea es sinónimo de estar disponible, sin entender que quizá sea razón de estar conectados por cuestiones académicas, laborales o de atención a alguna situación familiar específica. De este modo, sólo se per- cibe como un dispositivo de control a distancia "correa digital" estando todos al alcance de todos (Luz, 2010).

En voz de los entrevistados:

[...] Tiene que estar como que disponible la gente para mí y yo también.

[...] Soy una persona que está bastante localizable de inmediato.

[...] A mi mamá la estuvimos presionando entre todos: "por Dios, trae un celular; me desespera [...] que ella no traiga celular porque yo no puedo estarla checando, monitoreando, viendo a qué hora llega, esto el otro, entonces es como calcularle" [...] me desespera que no la pueda localizar.

Estamos ante un nuevo fenómeno de concebir la interacción interpersonal, siendo las redes sociales un nuevo medio para relacionarnos con quienes tenemos lejos y para alejarnos de quienes tenemos cerca. Podemos compartir un mismo espacio sin que en realidad exista una comunicación asertiva, donde la ausencia no es física sino mental, lo que rompe las dinámicas de relación despersonalizando la comunicación.

\section{Conclusiones}

La modificación en las relaciones interpersonales, en función del uso de redes sociales para su comunicación, trae consigo cambios significativos. El móvil, mediante el discurso de los informantes, fue significado sobre todo por el interés de acceder a las redes sociales porque desde una perspectiva utópica les permite mantener relaciones interpersonales donde la distancia y el tiempo no lo permiten, posibilitando la comunicación por encima de las disponibilidades; sin embargo, en la cotidianeidad ponen una barrera entre las relaciones que tienen cercanas, como lo es con la familia y los amigos, las cuales con el tiempo se ven deterioradas.

El crecimiento de esta dualidad nos hace parecer que siempre ha existido el uso de las tecnologías y que por tanto su desaparición y/o prohibición por el uso desmedido estarían fuera de contexto; sin embargo, falta recordar la infinidad de habilidades que se tenían antes de su aparición, desde recordar los números telefónicos de toda la familia dígito a digito, hacer uso de la lógica y de orientación para llegar a algún sitio sin el uso del GPS, ocupar horas que ahora dedicamos en redes sociales a actividades recreativas y, lo más importante, establecer relaciones interpersonales cercanas con una construcción del lenguaje por medio de los sentidos y la atención a la otra persona. 
El teléfono móvil es un dispositivo por el que pueden transitar cargas de contenido constructivo, que aporte habilidades y simplifique procesos, pero a su vez también puede ser portador de basura virtual que invita al ocio y a la segregación, contribuyendo al detrimento de otras actividades como leer, aprender, interactuar, jugar, etcétera. Hoy nuestra vida parece estar en la palma de nuestras manos, y no es incorrecto en tanto esta situación no rebase nuestra habilidad para relacionarnos, para utilizar la razón, la lógica, el tiempo en nuestra vida, sembrar a partir de su uso nuevo conocimiento y desechar lo negativo que nos pueda aportar.

Generar conciencia en la sociedad acerca de una comunicación asertiva dentro de las relaciones interpersonales es indispensable porque ello nos permitirá establecer vínculos eficaces en donde primen la calidad y la calidez.

\section{Sugerencias y limitaciones}

Estudios relacionados con el uso de las tecnologías y salud, y lo que se desprende de ellas, actualmente son atendidos desde otras perspectivas disciplinares; enfocarla desde la disciplina de enfermería nos permitirá establecer por medio de los hallazgos encontrados, programas preventivos relacionados con la salud física y mental. Para el desarrollo de este estudio no se tuvo limitación alguna.

\section{Referencias}

Aparicio, M. B, \& Zermeño, E. A. (2010). Relaciones familiares y nuevas tecnologías en el siglo XXI. Universidad de Navarra, 44-48. Recuperado de: http://bit.ly/2lah8hH

Álvarez-Gayou, J. L. (2005). Cómo hacer investigación cualitativa. Fundamentos y metodología. Barcelona: Paidós, pp. 187-193.

Antolinez, M. P., Rivero, A., \& Torres, L. (2013). El teléfono móvil: usos normas y conflictos familiares. Federación Española de Sociología, 15-17. Recuperado de: https://bit.ly/2HVqcdq

Castillo, E. (2000). La fenomenología interpretativa como alternativa apropiada para estudiar los fenómenos humanos. Investigación y educación en Enfermería, 1(18),
27-35. Recuperado de: https://bit.ly/2DNfu5H

Comisión Nacional de Bioética (1979). Informe Belmont: Principios y guías éticos para la protección de los sujetos humanos de investigación. [online] National Institutes of Health. Recuperado: http://bit.ly/2KlcSzO

Cornejo, M., \& Tapia, M. L. (2012). Redes sociales y relaciones interpersonales en internet. Fundamentos en Humanidades, 2(24), 221. Recuperado de: https://bit.ly/1yGZE2s

Fortunati, L., \& Manganelli, A. M. (2002). El teléfono móvil de los jóvenes. Estudios de juventud, 57(2), 59-78.

Giménez, M., \& Zirpoli, R. (2015). Trastornos psicológicos vinculados al uso del whats app. VII Congreso internacional de investigación y práctica profesional en psicología, XXII jornadas de investigación décimo encuentro de investigadores en psicología, p. 58.

Guillén, D. M., Beltrán, N. I., Flores, B., Aristizabal, G. P., \& Garduño, M. (2016). Problemas de salud derivados del uso del teléfono celular. Rev. iberoam. educ. investi. Enferm, 4(6), 62-70. Recuperado de: https://bit.ly/2KeseYr

Koepsell, D., \& Ruiz, M. (2015). Ética de la Investigación, Integridad Científica. México: Conbioetica, 123-167.

Lojo, G. (2014). Deconstrucción del lenguaje: habla escrita en el teléfono celular. Ciencias de la salud, 1(4), 14-19. Recuperado de: https://bit.ly/2JXzalX

Luz, A. (2010). El teléfono celular y las aproximaciones para su estudio. Nueva época, 14, 144-150. Recuperado de: https://bit.ly/2IVmUc6

Ortiz, G., Hegewisch, J., Palacios, M., \& Espinoza, N. (2016). Hábitos de los consumidores móviles en México 2017. México: Deloitte, 3-16. Recuperado de: http://bit.ly/2juKf3V.

Pedrero, E. J., Rodríguez, M. T., \& Ruiz, J. M. (2012). Adicción o abuso del teléfono móvil: Revisión de la literatura. Adicciones, 2(24), 140. https://doi.org/10.20882/adicciones.107

Richard, L. (2004). Book Review The mobile connection: The cell phone's impact on society. International Journal of Technology and Human Interaction. San Francisco: USA, pp. 101-104.

Rivero, F. (2017). Informe mobile en España y en el mundo. febrero 2018, de DITRENDIA Recuperado de: http://bit.ly/2ogkwQ1.

Rodríguez, G., Gil, J., \& García, E. (1999). Metodología de la investigación Cualitativa. Málaga: Aljibe, pp. 168-182.

Tamayo, J. (2017). Hábitos de Usuarios de Internet en México. Febrero 2018, de Web marketing. Recuperado de: http:// bit.ly/2AIFV9m 
Artículo Empírico | Redes sociales: Transformando la comunicación... | Guillén-Cadena eZ Reyes-García

\section{Meta-Análisis del Artículo}




\section{Dimensión Cuantitativa}

\section{Perfil de Evaluación entre pares}
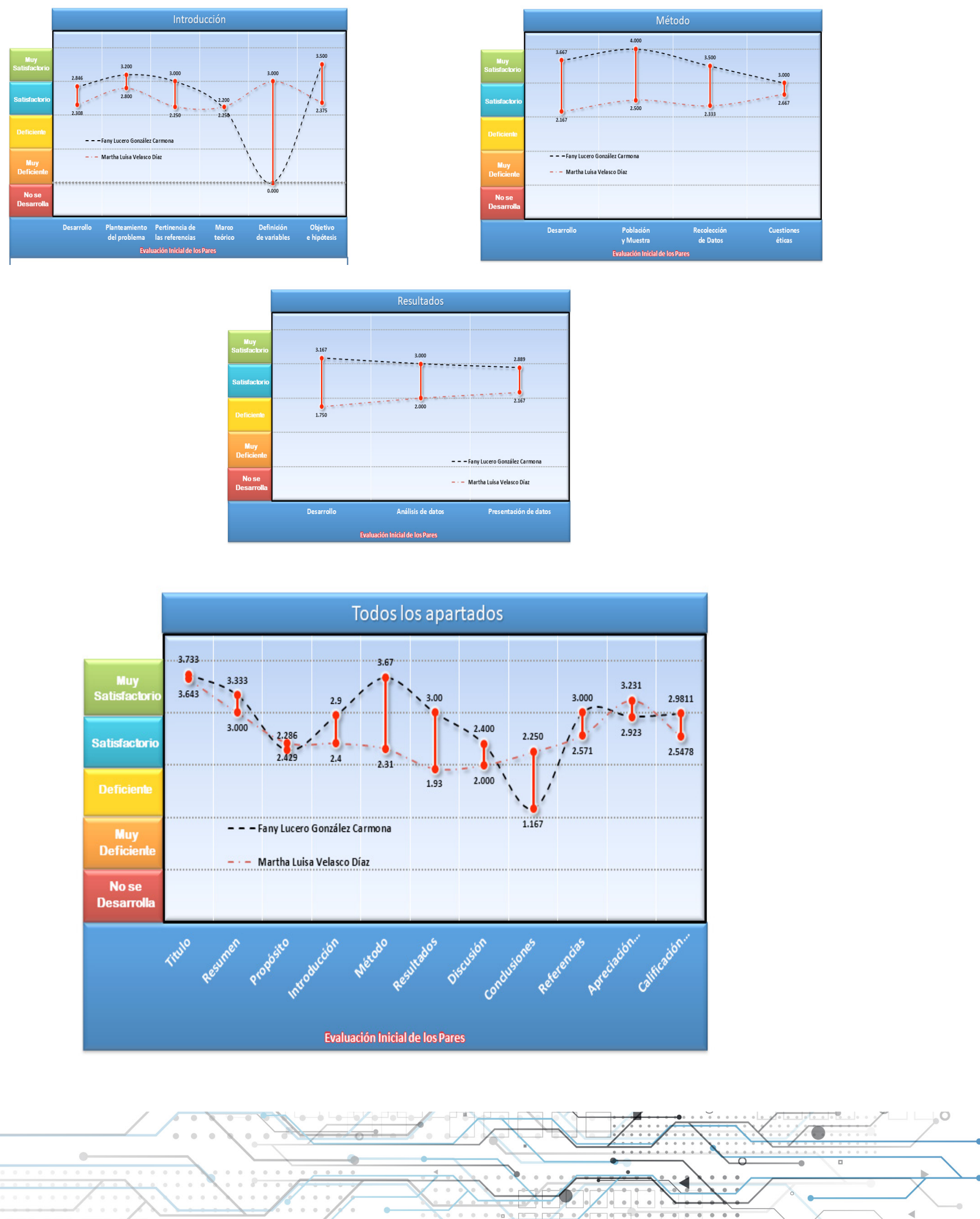
Artículo Empírico | Redes sociales: Transformando la comunicación... | Guillén-Cadena eZ Reyes-García

\section{Índice de Concordancia}

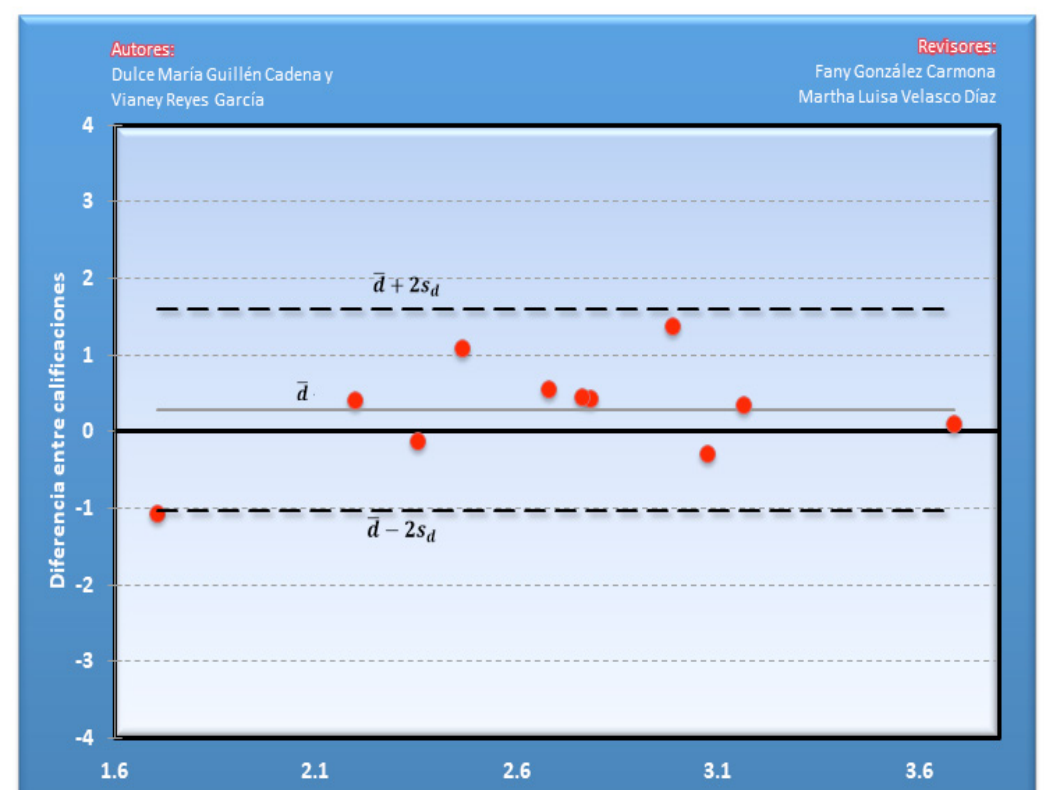

Índice de Acuerdo

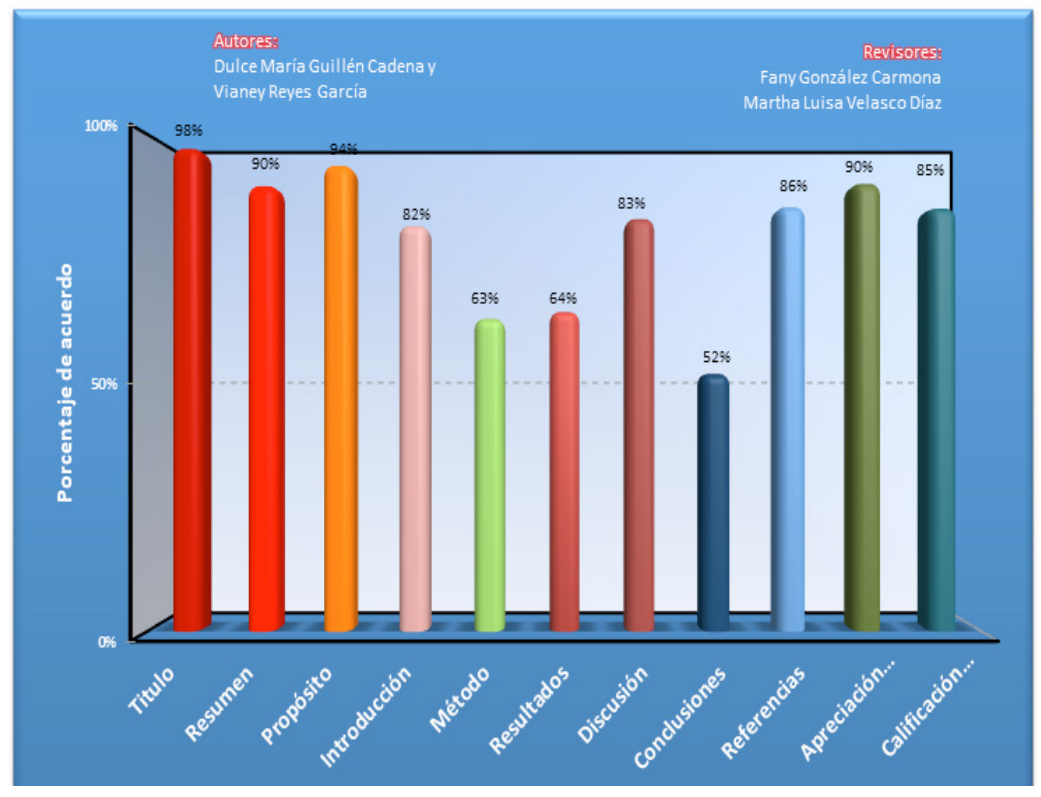




\begin{tabular}{|c|c|}
\hline Revisor 1 & Revisor 2 \\
\hline Fany Lucero González Carmona & Martha Luisa Velasco Díaz \\
\hline \multicolumn{2}{|c|}{ Título/Autoría } \\
\hline $\begin{array}{l}\text { En el título se hace referencia a la transformación de la } \\
\text { comunicación; sin embargo, en los hallazgos, más que } \\
\text { hablar de ese proceso de transformación se enfatiza en } \\
\text { cómo es la comunicación actual con base en el uso de } \\
\text { las redes sociales. Sugeriría por ello cambiar esta pala- } \\
\text { bra del título, la cual da la impresión de que se hablará } \\
\text { de todo este proceso de cambio entre el antes y el ahora } \\
\text { en la comunicación interpersonal. }\end{array}$ & El formato APA indica omitir el uso de gerundio \\
\hline \multicolumn{2}{|c|}{ Resumen } \\
\hline $\begin{array}{l}\text { Considero necesario señalar el número de participan- } \\
\text { tes y brevemente el procedimiento llevado a cabo, como } \\
\text { número de entrevistas realizadas. Las palabras clave, } \\
\text { aunque sean cinco en teoría, son solo tres compuestas } \\
\text { ya que "redes sociales" o "relaciones interpersonales" } \\
\text { cuentan como una sola. Una palabra clave más puede } \\
\text { ser referente a la población de estudio. }\end{array}$ & $\begin{array}{l}\text { Describir de manera breve y concreta las características } \\
\text { de la población Evitar el uso de gerundio Dar un renglón } \\
\text { para explicar las relaciones interpersonales }\end{array}$ \\
\hline \multicolumn{2}{|c|}{ Próposito del Estudio } \\
\hline $\begin{array}{l}\text { La bibliografía referenciada no guía directamente al pro- } \\
\text { pósito del estudio. Presentan más datos estadísticos so- } \\
\text { bre el uso de redes sociales y teléfonos móviles que son } \\
\text { sumamente necesarios e interesantes pero casi no hay } \\
\text { información propiamente sobre el tema de la comunica- } \\
\text { ción y las relaciones interpersonales. Considero necesa- } \\
\text { rio plantear las preguntas de la investigación y objetivos } \\
\text { específicos. Al señalar que es "a partir de la aparición de } \\
\text { las redes sociales..." apelaría al origen de dichas redes, se- } \\
\text { ría óptimo enfatizar que es a partir del uso constante de } \\
\text { las mismas, más que de su aparición propiamente }\end{array}$ & $\begin{array}{l}\text { Ampliar la explicación del objetivo del estudio, ya que } \\
\text { no queda claro cómo se va llegar a las conclusiones }\end{array}$ \\
\hline
\end{tabular}




\begin{tabular}{|l|}
\hline \multicolumn{1}{|c|}{ Revisor 1 } \\
\hline Sntrod \\
tas de investigación. Considero necesario nuevamente \\
enfatizar que no es a partir de la aparición de redes so- \\
ciales, sino con base en el uso continuo de las mismas. \\
Se señalan brevemente las aportaciones de Jerome Bru- \\
ner para hacer el análisis pero no se desarrolla propia- \\
mente un marco teórico, sería necesario desarrollarlo \\
más e incluirlo en el análisis. La información citada en \\
la introducción corresponde a datos estadísticos. Es ne- \\
cesario citar investigaciones ya realizadas en este mis- \\
mo campo de conocimiento, de modo que enmarquen \\
esta investigación, la necesidad de continuar indagan- \\
do, que se plantee el problema con precisión y se justi- \\
fique socialmente.
\end{tabular}

Revisor 2

ntroducción

\section{Método}

Agregar cifras de México de una referencia primaria.La cita de Rivero, 2017 es secundaria Definir, explicar las variables del estudio Redactar en tercera persona del singular Revisar un par de acentos del resumen e introducción Describir breve y concretamente entre paréntesis a que se refiere con los cambios frecuentes por el uso del celular

Considero necesario describir paso a paso cómo se llevó a cabo la recogida de datos, cómo inició el contacto con los participantes en la empresa señalada, cuántas observaciones se llevaron a cabo y qué espacios ocurrieron así como el número de entrevistas realizadas.

Describir el tipo de preguntas, el objetivo, de ser posible ponerlas en el apartado de apéndice Mencionar si es entrevista estructurada, semiestructurada En los aspectos éticos se abordan los principios del Informe Belmont pero no se explican como se aplicarían a la investigación. El procedimiento descrito en realidad es el diseño Los materiales son el procedimiento, por lo tanto revisar con detalle que va en la sección de procedimientos (describir paso a paso lo que se hizo para obtener los datos ) materiales(equipo, objetos que se utilizaron para obtener la información ) Explicar si dentro de las preguntas se hablaba de ser anónimo

\section{Resultados}

Es necesario redactar los resultados de modo que sean comprensibles las afirmaciones que se hacen desde la teoría, con lo obtenido en las entrevistas, ya que se inicia afirmando cierto cambio en la comunicación y posteriormente se colocan las voces de los entrevistados pero no están del todo vinculados, ni se explican unas con otras. Se presentan afirmaciones generales basadas en la teoría, pero es necesario presentar afirmaciones propias de esos participantes y de los cambios que permitieron ver. Las categorías que se presentan son repetitivas Si en el objetivo se considera el proceso de transformación de la comunicación, es necesario resaltar en estos hallazgos dichos cambios, el antes y el ahora.

La figura no cumple ninguna función En la descripción cualitativa de lo que dijeron las personas, hay que usar signos de admiración, comas, puntos, para que se entienda mejor el discurso 


\section{Revisor 1}

Revisor 2

\section{Discusión}

Se presenta la interpretación de los resultados con base en lo ya señalado en la investigación precedente pero no se discute con ellos en términos de divergencia o coincidencia. Es necesario realizar la interpretación propia de los resultados de modo que sea posible desarrollar una discusión

Es importante agregar una sección de sugerencias y limitaciones, retomar autores del marco teórico . Agrupar el tema de: “... poco afectiva” en una misma sección para que no suene repetitivo

\section{Conclusiones}

Es necesario incluir los apartado de las contribuciones de este estudio a las investigaciones previas, las deficiencias y las proponer futuras investigaciones. Considero importante concluir a la luz de los resultados de explicar el punto de vista de las autoras y notar si coincide forma específica más que señalar solo generalidades sobre el tema.

\section{Referencias}

Las referencias no están ordenadas en orden alfabético y no cumplen con los criterios particulares de Manual de estilo de la APA. Son 16 referencias. Hay referencias que no aparecen en las citas, que están incompletas o Las referencias según el formato APA tienen que estar en orden alfabético y en sangría francesa que no corresponden en año. 
Artículo Empírico | Redes sociales: Transformando la comunicación... | Guillén-Cadena eZ Reyes-García

Historia del Proceso EDITORIAL 